\title{
The cost of diabetes in Canada over 10 years: applying attributable health care costs to a diabetes incidence prediction model
}

\author{
Anja Bilandzic, MPH (1); Laura Rosella, PhD $(1,2,3)$
}

This article has been peer reviewed.

Tweet this article

\begin{abstract}
Introduction: Our objective was to estimate the future direct health care costs due to diabetes for a 10-year period in Canada using national survey data, a validated diabetes risk prediction tool and individual-level attributable cost estimates.

Methods: We used the Diabetes Population Risk Tool to predict the number of new diabetes cases in those aged 20 years and above over a 10-year period (to 2022), using 2011 and 2012 Canadian Community Health Survey data. We derived attributable costs due to diabetes from a propensity-matched case control study using the Ontario Diabetes Database and other administrative data. We calculated total costs by applying the respective attributable costs to the incident cases, accounting for sex, year of diagnosis and annual disease-specific mortality rates.
\end{abstract}

Results: The predicted 10-year risk of developing diabetes for the Canadian population in $2011 / 12$ was $9.98 \%$, corresponding to 2.16 million new cases. Total health care costs attributable to diabetes during this period were $\$ 7.55$ billion for females and $\$ 7.81$ billion for males ( $\$ 15.36$ billion total). Acute hospitalizations accounted for the greatest proportion of costs $(43.2 \%)$. A population intervention resulting in $5 \%$ body weight loss would save $\$ 2.03$ billion in health care costs. A $30 \%$ risk-reduction intervention aimed at individuals with the highest diabetes risk (i.e. the top $10 \%$ of the highest-risk group) would save $\$ 1.48$ billion.

Conclusion: Diabetes represents a heavy health care cost burden in Canada through to the year 2022. Our future cost calculation method can provide decision makers and planners with an accessible and transparent tool to predict future expenditures attributable to the disease and the corresponding cost savings associated with interventions.

Keywords: diabetes, economics, attributable cost, prediction model, incidence, Canada

\section{Introduction}

The management and prevention of diabetes remains a health priority in Canada. With approximately 1.96 million people living with diabetes, ${ }^{1}$ and with a growing number expected to develop the chronic condition in the future, considering widescale strategies to curb the disease is of great importance. In particular, diabetes presents a significant constraint on the
Canadian health care system. In 2008, it was estimated that the cost of hospital care, physician care and drugs for diabetes was $\$ 2.18$ billion. $^{2}$ Looking toward the future, the Canadian Diabetes Association has projected that the overall direct cost of diabetes will be $\$ 3.1$ billion in 2020 , based on 3.7 million prevalent cases predicted using a specially developed diabetes cost model. ${ }^{3}$ At the individual level, Goeree and colleagues have estimated that the

\section{Highlights}

- We created an accessible and transparent tool to help health decision makers calculate future diabetes costs.

- We predicted the number of new diabetes cases in Canada in those aged 20 years and above over the next 10 years (2011/12 to 2021/22) and linked this with actual individuallevel health care costs of diabetes.

- By 2022, 2.16 million new cases of diabetes are expected, corresponding to $\$ 15.36$ billion in health care costs related to diabetes.

- This tool can model various riskreduction interventions in the population; e.g. a $5 \%$ weight loss in the population would save $\$ 2.03$ billion and a $30 \%$ risk reduction in the group with the highest diabetes risk would save $\$ 1.48$ billion.

attributable cost per incident case of diabetes in Ontario is approximately $\$ 2930$ in the first year after diagnosis and \$1240 in following years. ${ }^{4}$ Recently, Rosella and colleagues expanded upon this work to include a greater number of direct costs in the province, and found that the mean attributable cost during eight years of follow-up was \$9731 for females and \$10 315 for males. ${ }^{5}$

While work has been done across Canada to estimate the future economic costs of diabetes, ${ }^{3,6}$ most cost estimates and models are complex, not transparent or not readily usable by health decision makers. With the goal of preventing diabetes, a tool that allows decision makers to

Author references:

1. Dalla Lana School of Public Health, University of Toronto, Toronto, Ontario, Canada

2. Institute for Clinical Evaluative Sciences, Toronto, Ontario, Canada

3. Public Health Ontario, Toronto, Ontario, Canada

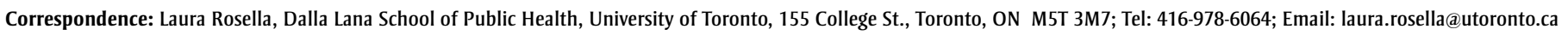


estimate the economic impact of future incident diabetes cases on the health care system would allow for more effective planning. From a program perspective, being able to quantify how actions today may shape case development and associated health care spending in the future is a considerable advantage in evaluating strategies. The objective of this study is, first, to estimate the future 10-year direct health care costs due to new diabetes cases in Canada using national survey data and individual-level attributable costs within the context of a diabetes risk prediction tool; and second, to apply the tool to two hypothetical intervention scenarios aimed at decreasing diabetes incidence in the population.

\section{Methods}

\section{Diabetes risk and incidence}

To estimate the predicted risk and number of new diabetes cases within the next 10 years, we used the Diabetes Population Risk Tool version 2.0. DPoRT 2.0 is an updated iteration of DPoRT, a predictive algorithm developed to calculate future population risk and incidence of physician-diagnosed diabetes in those aged 20 years and over. DPoRT was derived using national survey data individually linked to a chart-validated diabetes registry. This cohort was then used to create sex-specific survival models using baseline risk factors from the survey for diabetes incidence. Specifically, we assessed the probability of physician-diagnosed diabetes from the interview date until censoring for death or end of follow-up. The model was developed in the Ontario cohort and predictions from the model were validated against actual observed diabetes incidence in two external cohorts in Ontario and Manitoba. Variables used within its two sex-specific models include a combination of hypertension, ethnicity, education, immigrant status, body mass index, smoking status, heart disease and income. Full details on the model specification and validation can be found elsewhere. ${ }^{7}$ The regression model can run on nationally available population health surveys and has been updated (DPoRT 2.0) and used to established prevention targets for diabetes. ${ }^{8}$

For this study, we used DPoRT 2.0 to generate incidence predictions based on the recent 2011 and 2012 Canadian Community Health Survey (CCHS). The CCHS collects information on the demographics, health status and determinants of health of the Canadian population. It is a nationally representative survey that uses a crosssectional study design and is administered on an ongoing basis, with annual data reporting. It covers $98 \%$ of the population aged 12 years and older; exceptions include people living on Indian reserves and Crown lands, institutionalized residents, full-time members of the Canadian Forces and people living in particular remote regions. ${ }^{9}$ The sample size for this survey was 124 929; after applying exclusion factors (e.g. respondents aged under 20 years and those with existing diabetes were excluded), the final sample size used in analyses for this study was 90 631, representing 21598180 people when weighted.

\section{Intervention scenarios}

In addition to baseline estimates (i.e. all demographic and risk factors as outlined above), we ran two hypothetical intervention scenarios to examine how implementing interventions aimed at reducing diabetes risk would affect the incidence of the disease and the cost to the health care system.

First, we modelled a nontargeted intervention leading to an average 5\% weight loss in the population. A $5 \%$ drop in weight has a positive impact on glycemic and cardiovascular health clinically ${ }^{10}$ and represents a modest and realistic weight decrease for many. This intervention would reflect a large-scale change, such as a change in the built environment (e.g. it has been shown that populations in highly walkable areas have lower overweight and obesity prevalence rates ${ }^{11}$ ) or the implementation of improved nutrition labelling.

Second, we ran an intervention scenario in which those in the highest-risk decile (i.e. those who have a 10 -year risk of developing diabetes $\geq 22.6 \%$ ) were targeted for an intervention leading to a $30 \%$ reduction in their risk. For example, this approach might consist of a targeted lifestyle intervention program or a pharmaceutical intervention that has proven efficacy in randomized trials. ${ }^{12}$

\section{Attributable cost estimates}

To estimate future costs attributed to diabetes, we used results from a recent propensity-matched cohort study. ${ }^{5}$ Briefly, this study used the Ontario Diabetes
Database (ODD) to identify new cases of physician-diagnosed diabetes from 01 April, 2004, to 31 March, 2012. Three control subjects without diabetes were matched to each person with diabetes; they were hard matched on index date ( \pm 30 days), age ( \pm 90 days) and the logit of the propensity score. This score was the predicted probability of developing or not developing diabetes, calculated from a logistic regression consisting of age, rurality, comorbidity, geographic location and neighbourhood income quintile as predictive variables.

During this eight-year follow-up period, individual-level direct health care costs were tracked annually. These costs were extracted by linking various health care utilization databases and following a person-level costing methodology specifically developed and validated for Ontario administrative databases. ${ }^{13}$ These costs were from the perspective of the health care system, and included costs from inpatient hospitalizations, emergency department visits (ED), same-day surgeries (SDS), dialysis, oncology clinic visits, fee-for-service physician and non-physician services, nonfee-for-service physicians, prescription medications, laboratory, rehabilitation, complex continuing care, long-term care, mental health inpatient stays, home care services and medical devices. Attributable costs were calculated as the difference in cost between those with and without diabetes.

\section{Cost calculations}

We developed a cost calculator to use DPoRT 2.0 incidence predictions and perpatient attributable cost values to estimate the direct health care costs attributable to diabetes, over a future 10-year period. All calculations were sex-specific, reflecting differences in health care use $^{5}$ and perhaps self-care patterns. ${ }^{14}$ The number of incident cases projected to occur each year was multiplied by the corresponding per-patient annual cost, dependent on the time since the diabetes diagnosis, and taking into account annual mortality rates, which were generated from the agespecific mortality rates of patients in the ODD. Mortality rates were specific to year of follow-up. We assumed that deaths occurred halfway through the year, and as such, half of those who died contributed costs to that specific year. Because the individual costing estimates used eight years of follow-up in the analysis, it was assumed that the costs attributable to 
individuals who contributed costs in years 9 and 10 after diagnosis did so at the same monetary value as year 8 . As there was a downward tendency in health care costs observed for the first eight years, we conducted a sensitivity analysis whereby years 9 and 10 costs were estimated by following a linear trend to see the effect of changing the individual attributable costs on the resulting cost estimates.

\section{Cost distribution by sector}

In order to estimate the burden of costs by sector, the mean costs per health care segment over the eight years of follow-up were converted to percentages and multiplied by the total costs estimated from the cost calculator.

We performed all statistical analyses using SAS version 9.4 (SAS Institute Inc., Cary, NC, USA).

\section{Results}

The predicted 10-year risk of developing diabetes for the Canadian population as a whole is $9.98 \%$, corresponding to 2156000 new cases between 2011/12 and $2021 / 22$. The risk is higher among males than females $(11.23 \%$ vs. $8.85 \%)$, with males representing more new cases overall. The estimated total health care cost of these new cases is $\$ 15.36$ billion.

If a population-level (small impact and large reach) intervention was put in place that resulted in an average body weight loss of $5 \%$ in the population, the 10 -year predicted risk of developing diabetes would drop to $8.67 \%$, resulting in 1873000 cases developing in this time period (Table 1). This reduced number of new cases would cost $\$ 13.33$ billion, resulting in a savings of $\$ 2.03$ billion when compared with baseline characteristics.

In contrast, if an intervention targeting those with the highest predicted risk (the top $10 \%$ of the highest-risk group) in the population were carried out, the overall risk of developing diabetes would be $9.02 \%$. This would translate to 1949000 new cases, at a total cost of $\$ 13.88$ billion (Table 1). Compared with the baseline scenario, $\$ 1.48$ billion in direct health care costs would be averted.

When we estimated costs for years 9 and 10 using a linear trend based upon years 1 to 8 of observation, the results were not

TABLE 1

Health care costs attributable to diabetes, baseline scenario and two hypothetical intervention scenarios, Canada, both sexes, 2011/12 to 2021/22

\begin{tabular}{|c|c|c|c|c|}
\hline & & 10-year risk ${ }^{\mathrm{a}}(\%)$ & $\begin{array}{l}\text { Incidence (\# of } \\
\text { cases, thousands) }\end{array}$ & $\begin{array}{l}\text { 10-year overall cost } \\
\text { (\$, billions) }\end{array}$ \\
\hline \multicolumn{5}{|c|}{ Baseline characteristics } \\
\hline Overall & & 9.98 & 2156 & 15.36 \\
\hline \multirow{2}{*}{ Sex } & Female & 8.85 & 1000 & 7.55 \\
\hline & Male & 11.23 & 1156 & 7.81 \\
\hline \multicolumn{5}{|c|}{$5 \%$ weight loss in population } \\
\hline Overall & & 8.67 & 1873 & 13.33 \\
\hline \multirow{2}{*}{ Sex } & Female & 7.79 & 880 & 6.64 \\
\hline & Male & 9.64 & 993 & 6.70 \\
\hline \multicolumn{5}{|c|}{$30 \%$ risk reduction in highest-risk group } \\
\hline Overall & & 9.02 & 1949 & 13.88 \\
\hline \multirow{2}{*}{ Sex } & Female & 8.20 & 927 & 6.97 \\
\hline & Male & 9.93 & 1022 & 6.91 \\
\hline
\end{tabular}

Abbreviation: \$, Canadian dollars.

a 10-year risk of developing diabetes.

${ }^{\mathrm{b}}$ The highest-risk group has a 10-year risk of developing diabetes $\geq 22.6 \%$.

very different from estimates assuming equal costs for years 8,9 and 10 . Because the total difference was approximately $\$ 15.96$ million, we determined that using the originally proposed costing methodology was appropriate.

In terms of distribution of costs, the largest proportion of health care spending goes to acute hospitalizations: approximately $43.2 \%$ ( $\$ 6.64$ billion). The second largest share is for physician costs, which represent $21.9 \%$ ( $\$ 3.37$ billion) of all costs. Prescription medications and assistive devices account for $16.9 \%$ of costs ( $\$ 2.60$ billion); followed by home care, nonphysician care and long-term care ( $\$ 1.05$ billion); other inpatient services ( $\$ 0.88$ billion); and ED, SDS and outpatient clinic services ( $\$ 0.83$ billion) (Figure 1).

\section{Discussion}

Between 2011/12 and 2021/22, new cases of diabetes are estimated to result in $\$ 15.36$ billion in Canadian health care costs, almost two-thirds of which will be spent on acute hospitalizations and physician services $(65.1 \%)$. This study introduces a novel way of estimating future health care costs attributable to new cases of diabetes. The linkage of an incidence prediction model with individual-level attributable costs allows for estimates to be derived for different segments of the population, including sex-specific estimates, as well as region-specific costs. The ability to predict incident cases annually also allows users to calculate costs per year in the future and costs by year of follow-up for any number of years ranging from one to 10 .

Because this is a new cost methodology that focusses on the development of incident diabetes cases, it is difficult to compare these estimates with previously projected costs. Previous Canadian estimates have used varying health care costs associated with diabetes, and have either focussed on projected costs per year based on prevalent cases $^{3,6}$ or have retrospectively reported on cases that have already occurred. ${ }^{15-17}$ The report Economic Burden of Illness in Canada, 2005-2008 (EBIC) offers comprehensive cost estimates for a variety of conditions, including diabetes. ${ }^{2}$ Our cost methodology differs from that used in EBIC in that EBIC used prevalencebased costs while we used incidencebased costs. In addition, we estimated attributable costs; our costs represent the difference in health care costs that are directly attributable to diabetes, while EBIC only generates overall cost of illness. This is achieved by using a propensitymatched cohort design. ${ }^{5}$ Finally, EBIC did not couple these estimates with predictions on future cases and therefore did not 
FIGURE 1

Distribution of total 10-year direct health care costs attributable to diabetes ( $\$$ billions), Canada, 2011/12 to 2021/22

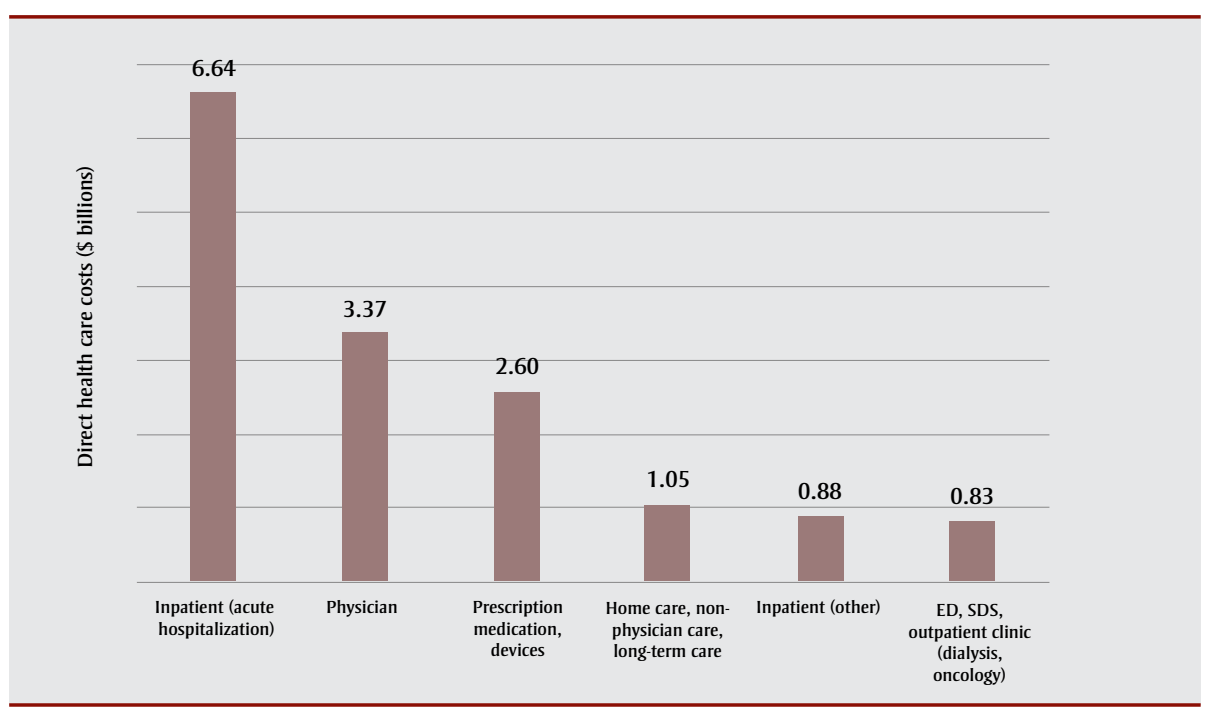

Abbreviations: ED, emergency department visits; SDS, same-day surgery.

Note: Figures have been rounded.

allow for intervention planning or estimates on future cost burden.

\section{Strengths and limitations}

This methodology has unique strengths. First, the costs are based on actual observed health care cost data from a prospective cohort over eight years of observation. Therefore, these are not projected estimates only, but instead reflect the reality of contemporary diabetes costs to the health care system. The use of attributable cost as a metric is also advantageous as it represents the excess cost of disease beyond average spending, due to the comparison with the group without the disease. Using total costs based only on the diseased population can overestimate the spending on disease and can provide inflated evaluations. ${ }^{2}$

Second, this method is simple to apply and can be used by a variety of end users. This is the aim of the tool itself-to be accessible and transparent for use within applied settings, such as provincial ministries of health and regional health bodies. Being able to model intervention scenarios, unique to the user's program goals and region, is an added benefit for health planners and decision makers who seek to estimate the economic offsets of various diabetes prevention strategies. Being able to estimate the cost averted, in addition to the number of cases prevented through customized intervention strategies, allows for the evaluation of different policy options and can assist in determining how best to move forward with chronic disease prevention activities. For example, in Canada, there are dozens of promising policy choices and interventions aimed at healthy living being led through federal, provincial and regional partnerships. ${ }^{18}$ Such programs could benefit from a tool that would factor in context-specific population characteristics to evaluate the most appropriate and feasible intervention strategies from an economic and health include providing information on the outcomes of improved treatment and disease management strategies. Since these approaches can lengthen life and possibly reduce costs, this information, combined with the effect on incidence, could offer insight into the combination of both treatment and prevention approaches.

The simplicity of this model does mean that several assumptions had to be made and must be acknowledged. First, the cost estimates are derived from a study that was based on Ontario data and thus the attributable costs used for national estimates assume that health care spending is similar in other provinces and territories. However, it is known that differences exist across jurisdictions, including within the as well as in provincial coverage for services and products such as medications and assistive devices. ${ }^{19,20}$ If province-specific perspective. Further applications could general care and management of diabetes, ${ }^{17}$ attributable cost estimates did become available in the future, the cost calculation method could easily be adapted to include these region-specific costs.

Second, this method uses average attributable costs by sex and year of follow-up. As such, it cannot account for costs averted within specific subgroups, who may be using more or less health care than the average. For example, in an intervention aimed at a high-risk group, it is likely that these people spend more health care dollars than the average, but their averted cost calculated will not reflect this (i.e. it will be underestimated using this method). Efforts to produce estimates that are defined to more specific populations would enable more accurate estimates, particularly when modelling intervention scenarios for certain target groups.

Third, the model does not account for future changes in health care spending or inflation. It is assumed that diabetes case management will remain the same through 2022 and that current models of care will continue to be applied and used in the same way. Given the window of 10 years, this assumption is likely appropriate. Longer prediction periods would need to address potential changes to care and management.

Finally, our estimates do not account for the costs associated with diabetes that are not related to health care, including indirect costs, out-of-pocket costs and costs not captured in administrative databases, as well as emotional and social costs for patients and other caregivers. It is estimated that direct health care costs only account for $17 \%$ of total costs attributable to diabetes, ${ }^{3}$ so it is crucial to consider these additional expenses in future research.

\section{Conclusion}

The goal of this work is to provide health decision makers with a readily usable tool that will allow them to make cost estimations up to 10 years in the future. Health planners and policy makers who focus on preventing diabetes at the population level can use this tool to evaluate different intervention strategies with customized incidence and cost predictions, which will assist them in determining the most appropriate actions for the future. 


\section{Acknowledgements}

Dr. Laura Rosella is supported by a Canada Research Chair in Population Health Analytics. This work was supported by the Canadian Institutes of Health Research Operating Grant from the Institute of Nutrition, Metabolism and Diabetes (funding reference number 126615). The funders had no direct role in the analysis, interpretation, writing or submission of this manuscript.

\section{Conflicts of interest}

The authors disclose no conflict of interest.

\section{Authors' contributions}

LR conceived the manuscript. LR and $A B$ contributed to analytic plan and interpretation. $A B$ prepared data and ran all analyses. $A B$ drafted the manuscript and LR edited and critically reviewed the final content.

\section{References}

1. Statistics Canada. CANSIM database: Table 105-0501: Diabetes, by age group and sex [Internet]. Ottawa (ON): Statistics Canada; 2014 [modified 2016 Apr 21; cited 2015 May 01]. Available from: http://www5.statcan.gc.ca /cansim/a26?lang = eng\&id $=1050501$

2. Public Health Agency of Canada. Economic burden of illness in Canada, 2005-2008. Ottawa (ON): Public Health Agency of Canada; 2014 [Catalogue No.: HP50-1/2013E-PDF].

3. Somerville R. An economic tsunami: the cost of diabetes in Canada. Toronto (ON): Canadian Diabetes Association; 2009.

4. Goeree R, Lim ME, Hopkins R, et al. Prevalence, total and excess costs of diabetes and related complications in Ontario, Canada. Can J Diabetes. 2009; 33(1):35-45.

5. Rosella LC, Lebenbaum M, Fitzpatrick $\mathrm{T}$, et al. Impact of diabetes on healthcare costs in a population-based cohort: a cost analysis. Diabet Med. 2016; 33(3):395-403.

6. Ohinmaa A, Jacobs P, Simpson S, Johnson JA. The projection of prevalence and cost of diabetes in Canada: 2000 to 2016. Can J Diabetes. 2004; 28(2):1-8.
7. Rosella LC, Manuel DG, Burchill C, Stukel TA, PHIAT-DM team. A population-based risk algorithm for the development of diabetes: development and validation of the Diabetes Population Risk Tool (DPoRT). J Epidemiol Community Health. 2011; 65(7):613-20.

8. Rosella LC, Lebenbaum M, Li Y, Wang J, Manuel DG. Risk distribution and its influence on the population targets for diabetes prevention. Prev Med. 2014;58:17-21.

9. Statistics Canada. Canadian Community Health Survey user guide: 2012 and 2011-2012 microdata files. Ottawa (ON): Statistics Canada; 2013.

10. Canadian Diabetes Association Clinical Practice Guidelines Expert Committee. Canadian Diabetes Association 2013 clinical practice guidelines for the prevention and management of diabetes in Canada. Can J Diabetes. 2013;37(suppl 1):S1-S212.

11. Glazier RH, Creatore MI, Weyman JT, et al. Density, destinations or both? A comparison of measures of walkability in relation to transportation behaviors, obesity and diabetes in Toronto, Canada. PLoS One. 2014;9(1):e85295. Erratum in PLoS One. 2014;9(3): e91485.

12. Gillies CL, Abrams KR, Lambert PC, et al. Pharmacological and lifestyle interventions to prevent or delay type 2 diabetes in people with impaired glucose tolerance: systematic review and meta-analysis. BMJ. 2007; 334(7588):299.

13. Wodchis WP, Bushmeneva K, Nikitovic M, McKillop I. Guidelines on personlevel costing using administrative databases in Ontario. Toronto $(\mathrm{ON})$ : Health System Performance Research Network; 2013.

14. De Melo M, de Sa E, Gucciardi E. Exploring differences in Canadian adult men and women with diabetes management: results from the Canadian Community Health Survey. BMC Public Health. 2013;13(1):1089.

15. Pohar SL, Majumdar SR, Johnson JA. Health care costs and mortality for Canadian urban and rural patients with diabetes: population-based trends from 1993-2001. Clin Ther. 2007;29 Spec No:1316-24.
16. Dawson KG, Gomes D, Gerstein H, Blanchard JF, Kahler KH. The economic cost of diabetes in Canada, 1998. Diabetes Care. 2002;25(8):1303-7.

17. Simpson $\mathrm{SH}$, Corabian $\mathrm{P}$, Jacobs $\mathrm{P}$, Johnson JA. The cost of major comorbidity in people with diabetes mellitus. CMAJ. 2003;168(13):1661-7.

18. Pan-Canadian Public Health Network. Towards a healthier Canada - 2015 progress report on advancing the federal / provincial / territorial framework on healthy weights. Available from: www.phn-rsp.ca/thcpr-vcpsre -2015/index-eng.php\#initiatives

19. Canadian Diabetes Association, Diabète Québec. Diabetes: Canada at the tipping point: charting a new path. Toronto (ON): Canadian Diabetes Association and Diabète Québec; 2011.

20. Canadian Diabetes Association. The burden of out-of-pocket costs for Canadians with diabetes [Internet]. Toronto (ON): Canadian Diabetes Association; [date unknown; cited 2015 Jun 15]. Available from: http:// www.diabetes.ca/CDA/media /documents/publications-and -newsletters/advocacy-reports/burden -of-out-of-pocket-costs-for-canadians -with-diabetes.pdf 